DOI 10.31489/2020No2/96-99

UDC 621.7.537

\title{
ELECTRIC PULSE METHOD FOR PRODUCING A SMALL-DISPERSED PRODUCT OF COAL-WATER FUEL
}

\author{
Khassenov A.K. ${ }^{1}$, Nussupbekov U.B. ${ }^{1}$, Karabekova D.Zh. ${ }^{1}$, Stoev M. ${ }^{2}$, Bolatbekova M.M. \\ 1E.A. Buketov Karaganda University, Karaganda, Kazakhstan, ayanbergen@mail.ru \\ ${ }^{2}$ Neofit Rilski South-West University, Blagoevgrad, Bulgaria
}

\begin{abstract}
One of the most pressing issues is the effective use of coal combustion in the form of water-coal fuel, which has a number of advantages over layer or pulverized combustion. Coal-water fuel has a number of economic, operational and environmental advantages. The article considers the electric pulse method as a source of obtaining a fine product of coal-water fuel. The proposed method for grinding coal is based on the use of the energy of a pulsed shock wave resulting from a spark electric discharge in a liquid. The parameters of the electric pulse discharge for obtaining the required granulometric composition of the components of coal-water fuel are determined.
\end{abstract}

Keywords: electric pulse, electrical discharge,coal-water fuel, coal-water suspension, number of discharge, condenser capacity

\section{Introduction}

Coal-water fuel (CWF) has great prospects among alternative fuels. It is a liquid mixture of two or more components in different proportions, one of the proportions is a combustible element (coal and brown coal, peat, waste coal, petroleum coke, etc.).The use of water-coal fuel and its subspecies as an energy carrier in the heat power industry has a number of advantages in comparison with other methods of coal preparation and combustion. The main advantage is the reduction of losses during coal transportation, convenience of storage and intra - station movement, and the supply of CWF to the furnaces of power plants. Coal-water fuel is a promising fuel for replacing scarce and expensive fuel oil at energy facilities [1].

In thermal power plants, the efficiency of using coal-water fuel (CWF) significantly depends on its technological parameters: viscosity, sedimentation stability, granulometric composition of the solid phase, and calorific value [1]. The granulometric composition of the solid phase of coal-water fuel is primarily determined by the requirements of consumers. Depending on the use of water-coal fuel, the optimal parameters of solid particles for coal-fired TPP are $250 \mu \mathrm{m}$, for fuel-oil TPP $150 \mu \mathrm{m}$, for boilers $45 \mu \mathrm{m}$, for internal combustion engines $25 \mu \mathrm{m}[2,3]$. The main task of water-coal fuel is coal grinding, which must be solved in the production process. The technology for producing coal-water fuel consists in grinding coal to a fine state and activating components (chemical additives) that are added to increase the fluidity of the watercoal suspension (CWF), prevent stratification and give it stability. For coal grinding, ball and rod mills are used in several stages of dry or wet grinding, which are characterized by multi-stage processing, high metal consumption, high specific energy costs for grinding one ton of coal, low efficiency and significant capital expenditures in the construction of coal-water fuel preparation complexes $[4,5]$. In this regard, research work was carried out to obtain coal powder using electric pulse technology. The method for solid fuel grinding is based on the electrohydraulic effect method, which is designed to create shock waves in a liquid medium [6-8]. The peculiarity of the processing method is carried out inside the volume of liquid that is located in an open (closed) vessel, a specially formed pulsed electric (spark, brush and other forms) discharge around the zone of its formation, ultra-high hydraulic pressures arise that can perform useful mechanical work and are accompanied by a complex of physical and chemical phenomena.

The electrohydraulic effect is associated with a relatively slow energy accumulation in the power source and almost instantaneous release in the liquid medium. The main factors of the electro-hydraulic effect are high and ultra-high pulsed hydraulic pressures, which lead to the appearance of shock waves with sound and supersonic speeds. These factors have a variety of physical and chemical effects placed in it on a liquid or 
other objects. Thus, shock displacements of liquid that occur during the development and collapse of cavitation cavities can destroy non-metallic materials and cause plastic deformations of metal objects placed near the discharge zone [6].

\section{Experimental part}

The latest achievements of electronic and high-voltage pulse technology can dramatically reduce the size of electrical elements, significantly increase the reliability of various electrophysical installations. Adaptation to the conditions of electric pulse technologies of electrical equipment mastered in related electrophysical research and technological applications will make it possible to make a technical breakthrough in the production use of electric pulse technologies. The modern element base already opens up prospects for the creation and wide development of EI technologies in special applications with limited productivity. Further improvement of the plants in the direction of increasing their capacity will consistently involve other technological directions of the method in production use [9].

Unlike mechanical crushers, the electrohydraulic unit does not have much moving parts, it is made of ordinary structural steel, so the body practically does not wear out during operation. There is no mechanical contamination of the product with hardware metal, lining material and grinding media. The ability of the method to simply and widely change the nature of dynamic loading allows you to adjust the granulometric composition of the grinding product, to obtain a narrower class of product size than is possible with mechanical grinding. In addition, the electric pulse method has a number of advantages compared to known grinding methods, mainly due to the small dimensions and mobility of the equipment, as well as the absence of restrictions on the strength of the materials being destroyed [10].

The electric pulse installation consists of a power supply-control block, a storage capacitor, an air converter and working channel. The power supply-control unit allows you to control the operating mode of the unit (adjust the power supply current, turn the unit on and off). High-voltage pulse capacitors (rated voltage $100 \mathrm{kV}$ ), which perform the function of storing electricity and are designed to operate in a mode in which charges and discharges quickly replace each other, were used as energy storage devices (capacitors). Figure 1 shows a General view of the working channel of the electric pulse unit. A spark pulse discharge is formed in a liquid medium between a rod metal electrode and the base (bottom) of a steel cylindrical vessel.

As a working medium, industrial water was used, which is cost-effective and affordable. After treatment with electric pulse discharges to separate the coal powder from the water, the water mixture was first settled, then the finished product was separated from the water (filtered) and dried at a temperature of $20{ }^{\circ} \mathrm{C}$.

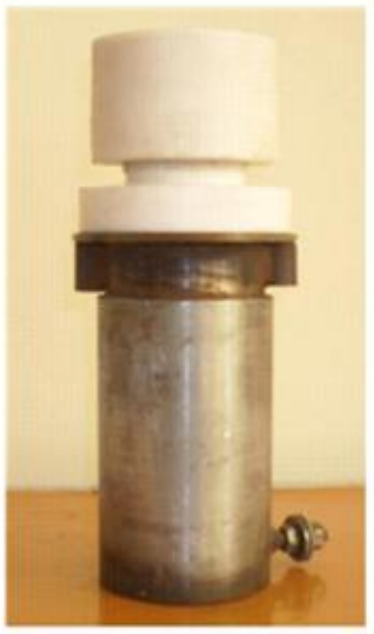

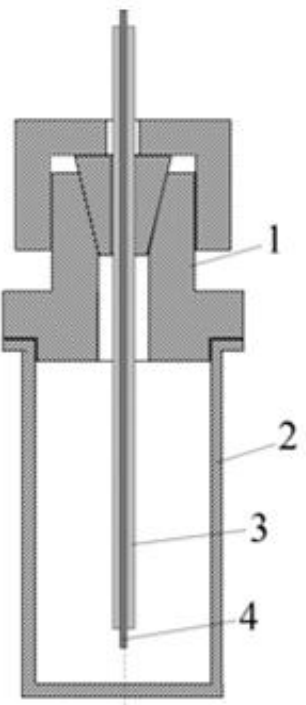

$\mathrm{b}$

Fig.1. Working channel of the electric pulse installation: a) main view; b) scheme;

1-cover made of fluoroplast, 2 - metal cylindrical container, 3-electrode insulation, 4-metal rod electrode

The capacitance of the capacitor (C) used for grinding raw materials was $0.25 \mu \mathrm{F}$ and $0.4 \mu \mathrm{F}$, the operating voltage of the discharge $\mathrm{U}=30 \mathrm{kV}$ and the number of pulse discharges $400,800,1200$. For carrying out research works, the coal of the Kuznetsk section was used, which is used as a lean additive to 
fatty coals. The average diameter of the initial coal fraction was $\mathrm{d}_{0}:=5 ; 10 ; 15 ; 20 \mathrm{~mm}$. Based on the results of processing coal with these parameters of the electric pulse unit, the granulometric composition of the product was determined on standard sieves and powders with a diameter (d) of $150 \mu \mathrm{m}$ and $250 \mu \mathrm{m}$ were sorted.

\section{Discussion of results}

Figure 2 shows the degree of coal grinding depending on the parameters of the electric pulse installation. The results of electric pulse crushing of coal can be seen that increasing the battery capacity of the capacitor $(0.25 \mu \mathrm{F}-0.4 \mu \mathrm{F})$ and increasing the amount of discharge (from 400 to 1200) the degree of grinding of raw materials increased.

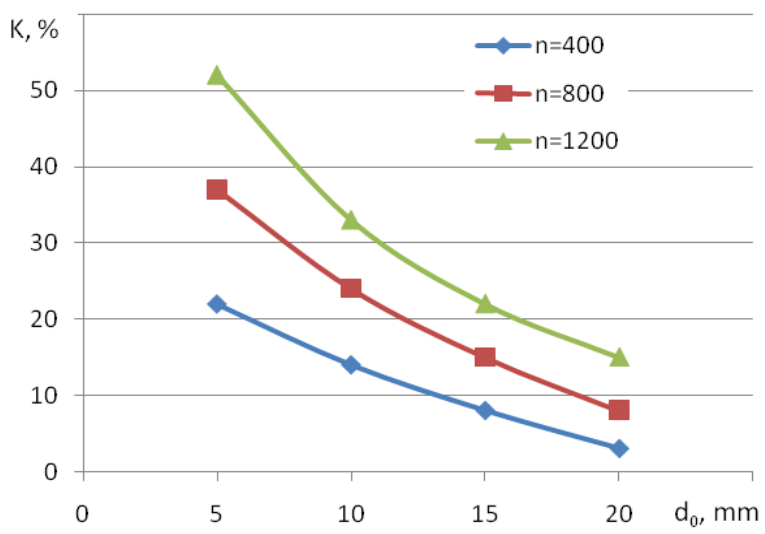

a) $C=0.25 \mu \mathrm{F}, \mathrm{d}=150 \mu \mathrm{m}$

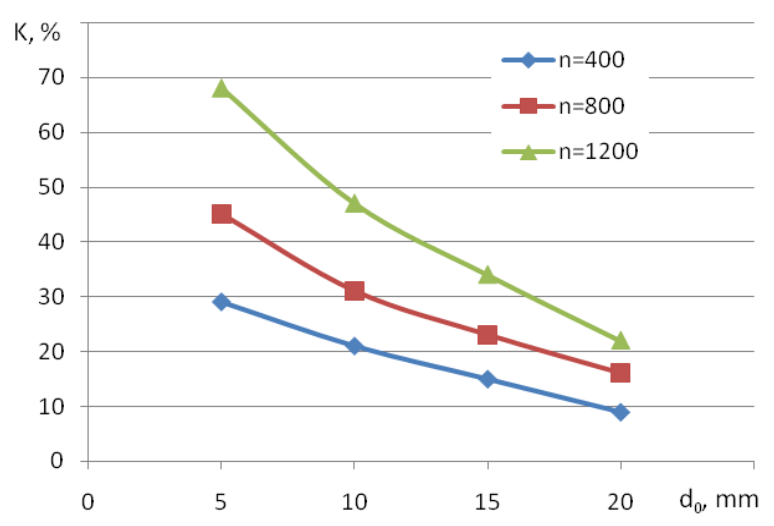

c) $C=0.25 \mu \mathrm{F}, \mathrm{d}=250 \mu \mathrm{m}$

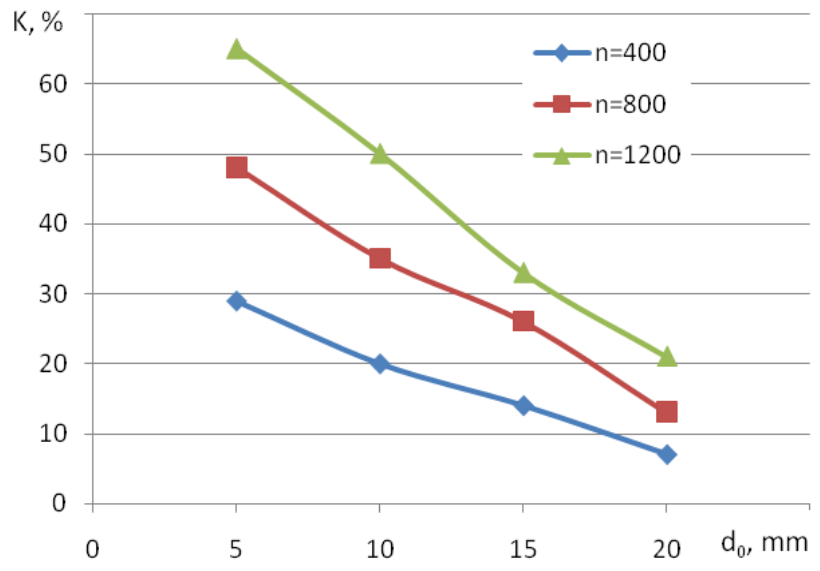

b) $C=0.4 \mu \mathrm{F}, \mathrm{d}=150 \mu \mathrm{m}$

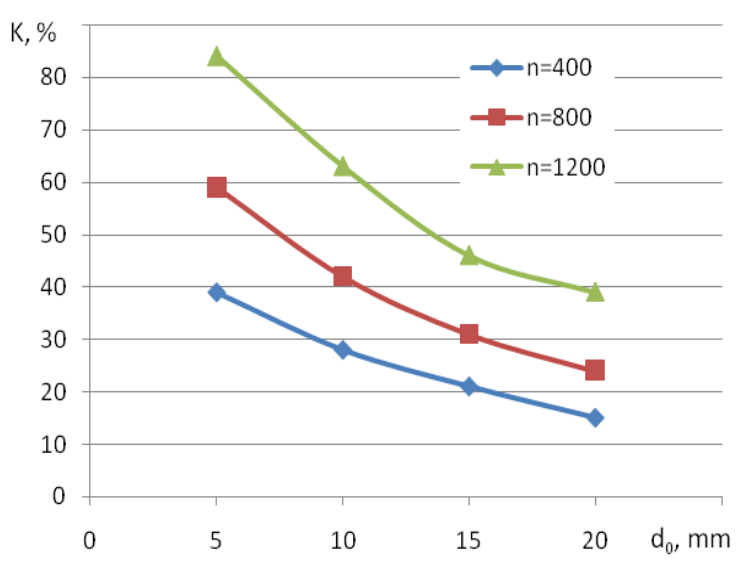

d) $C=0.4 \mu \mathrm{F}, \mathrm{d}=250 \mu \mathrm{m}$

Fig.2. Degree of coal grinding depending on the capacitance of the capacitor and the number of pulse discharges.

The degree of grinding of raw materials in the production of powder with a fraction diameter of 150 microns was the following interval: for coal with a diameter of the initial fraction of $5 \mathrm{~mm}-20-52 \%$, for 10 mm-14-32 \%, for $15 \mathrm{~mm}-8-22 \%$, for $20 \mathrm{~mm}-3-15 \%$ (figure 2, a). An increase in the degree of grinding of raw materials, depending on the given parameters of the electric pulse unit, was also observed in the production of coal powder with a diameter of 250 microns (figure $2, \mathrm{c}$ ).

The data shown in figure 2 are obtained from the average values of experiments repeated many times (5 times) during the treatment of coal by the electric pulse method. After each grinding operation, the diameter of the fraction of the resulting product was determined using the above-mentioned special standard sieves (GOST R 51568-99 (ISO 3310-1-90) laboratory Sieves made of metal wire mesh). From the results obtained, it can be seen that the degree of grinding of raw materials with an initial fraction diameter of $5 \mathrm{~mm}$ is higher than that of raw materials with a fraction diameter of 10-20 $\mathrm{mm}$. This is due to the fact that the spark gives 
energy to its surface, and therefore, the denser the material surrounds the discharge zone (the more crushed it is), the more efficiently and fully the energy released by the discharge channel is consumed. The main factors affecting the grinding mechanism are the intensity of the pressure wave pulse, its duration, the nature of the energy input in the discharge channel, the total duration of the grinding process, high-speed fluid flows formed as a result of volumetric micro-cavitation.

\section{Conclusion}

Research work on coal grinding by electric pulse method was carried out. According to the results of scientific work, a product with a diameter of fractions of $150 \mu \mathrm{m}$ and $250 \mu \mathrm{m}$, necessary for the manufacture of coal-water fuel, was obtained. The results of experimental work have shown that with the number of pulse discharges over 800 and the capacitance of the capacitor Bank of 0.4 UF (while the operating voltage is stable $-30 \mathrm{kV}$ ), solid fuel can be crushed to the required size. Currently, electric pulse technologies are used in the processes of cleaning heat exchangers from scale, crushing and grinding of natural ores, as well as processing of solid household waste. Carrying out these studies and implementing their results in enterprises will contribute to technological progress in the industry.

\section{REFERENCES}

1 Serant F.A., Tsepenok A.I., Ovchinnikov Yu.V., Lutsenko S.V., Karpov E.G. Preparation of coal-water fuel and its combustion technologies. Modern science: research, ideas, results, technologies. 2011, Vol. 1(6), pp. 95-101. [in Russian]

2 Samoylik V.G. Main technological parameters of coal-water fuel. Dressing of minerals Fig Kopalin. 2015, Vol. 61 (102), pp. 58-65. 45.

3 Khodakov, G.S. Water-coal suspensions in power engineering. Heat power engineering. 2007, Vol. 1, pp. 35-

4 Rizun A.R., GolenYu.V., Denisyuk T. D., Kononov V. Yu. Development and implementation of electric discharge process of disintegration of water-coal fuel components. Science of innovation. 2009, Vol. 5, Vol. 5. pp. 4246.

5 Zaydenberg V. E., Trubetskoy K. N., Murko V. I., Nekhoroshikh I. H. Production and use of water-coal fuel. 2001. M., Academyofmining Sciences, 176 p.

6 Yutkin L. A. Electrohydraulic effect and its application in industry: textbook. 1986, Leningrad, Mashinostroenie, $253 \mathrm{p}$.

7 Nussupbekov B.R., Khassenov A.K., Karabekova D.Zh., Nussupbekov U.B., Stoev M., Bolatbekova M.M. Coal pulverization by electric pulse method for water-coal fuel. Bulletin of the University of Karaganda-Physics. 2019, Vol. 4(96), pp. $80-84$.

8 Kuritnik I., Nussupbekov B.R., Khassenov A.K., Karabekova D.Zh. Disintegration of copper ores by electric pulses. Archives of Metallurgy and Materials. 2015, Vol. 60, 4, pp. 2449-2551.

9 Potokin A. S. Achievements and tasks of improving the electric pulse method of destruction of materials in connection with the assessment of prospects for its application in technologies of mining and processing of mineral raw materials. Intellectual potential of the XXI century: Stages of knowledge. 2011, Vol. 8, pp. 185-189.

10 Kosenkov V. M., Rizun A. R. Determination of the characteristics of pulsed destruction of coal using the Kola method. Electronic processing of materials. 2011, Vol. 2, pp. 100-107. 